\title{
Antibiotic Susceptibility of Candida Isolates in a tertiary care hospital in Southern India
}

Dear Editor,

Systemic infections due to yeasts and resistance to antifungals is on the rise in Indian hospitals. ${ }^{1}$ Candida albicans accounts for $40-60 \%$ of yeasts isolated in developed countries ${ }^{2}$ whereas Indian reports show an increased predominance of non $C$. albicans isolates. ${ }^{1}$ Increasing resistance to azoles and amphotericin B has been reported both from India and other countries. ${ }^{1,2}$ Given the paucity of data from southern India on the profile of yeast isolates and prevailing patterns of susceptibility to antifungals we speciated 77 yeasts obtained from culture of clinical specimens [Respiratory 22 (27.8\%), blood $22(25.3 \%)$, vaginal $12(15.2 \%)$, urine $13(16.5 \%)$ and others $10(12.7 \%)$ ] and estimated susceptibility to antifungal drugs. The study period was from September 2004 to March 2005. Isolates of Candida were identified by Gram stain of colony morphotypes suggestive of yeasts and were presumptively reported as $C$. albicans or Candida spp. based on the positive or negative germ tube test. Species level identification and antifungal susceptibility were done using the API ID 32C for identification and ATB Fungus 2 (bioMerieux, France). ${ }^{3}$ Manufacturers' instructions were strictly followed and adequate quality control measures were undertaken to ensure sterility and efficacy of media and reagents used.

The distribution of Candida spp. and their susceptibility is portrayed in the table. The common isolates were $C$. albicans 36(47\%), Candida tropicalis 18(23\%) and Candida pelliculosa 5(6\%). These three species accounted for $76 \%$ of Candida isolates. C. pelliculosa was isolated only from blood cultures of newborn in the neonatal ICU.

Germ tube test was positive in $89 \%$ of isolates speciated as $C$. albicans and $5 \%$ of $C$. tropicalis. The loss of susceptibility of $C$. albicans to azoles (fluconazole $-30 \%$ and itraconazole $75 \%$ ) is of concern although amphotericin B and flucytosine continue to show good efficacy. It is necessary to start presumptive therapy with either of these two antifungals when $C$. albicans is reported. Of the other Candida species only $C$. pelliculosa showed $100 \%$ susceptibility to the azoles. Flucytosine displays no efficacy against $C$. pelliculosa, $C$. krusei and $C$. parapsilosis. With multiple antifungals and varying susceptibility patterns of Candida it has now become necessary to perform antifungal susceptibility testing real-time and make reports available to the clinician for effective therapeutic outcome.

\section{References}

1. Chakrabarti A, Mohan B, Shrivastava SK, Marak RS, Ghosh A, Ray P. Change in distribution and antifungal susceptibility of Candida species isolated from candidaemia cases in a tertiary care centre during 1996-2000. Indian J Med Res 2002;116:5-12.

Table: Profile of Candida isolates and their susceptibility

\begin{tabular}{lccccccc}
\hline Isolate & Number $(\%)$ & FCNZ $(\%$ S) & ICNZ $(\%$ S) & AMPB $(\%$ S) & FLUCY $(\%$ S $)$ & GT $(+)(\%)$ & GT $(-)(\%)$ \\
\hline Candida albicans & $36(47)$ & 30.6 & 75 & 100 & 97.2 & 89 & 11 \\
Candida tropicalis & $18(23)$ & 22.2 & 77.8 & 88.9 & 72 & 5 & 95 \\
Candida pelliculosa & $5(6)$ & 100 & 100 & 60 & 0 & - & 100 \\
Candida krusei & $7(9)$ & 0 & 14.3 & 42.9 & 0 & - & 100 \\
Candida parapsilosis & $4(5)$ & 0 & 75 & 100 & 0 & - & 100 \\
Germ tube (-) isolates & $45(57.7)$ & 29.5 & 65.9 & 79.5 & 59.1 & 97 & \\
Germ tube (+) isolates & $33(42.3)$ & 27.3 & 78.8 & 100 & & \\
\hline
\end{tabular}

FCNZ- Fluconazole, ICNZ - Itraconazole, AMPB - Amphotericin B, FLUCY - Flucytosine, GT - Germ Tube 
2. Pfaller MA, Diekema DJ, Jones RN, Messer SA, Hollis RJ. Trends in antifungal susceptibility of Candida spp. isolated from pediatric and adult patients with bloodstream infections: SENTRY Antimicrobial Surveillance Program, 1997 to 2000. J Clin Microbiol 2002;40:852-6.

3. Buchaille L, Freydiere AM, Guinet R, Gille Y. Evaluation of six commercial systems for identification of medically important yeasts. Eur J Clin Microbiol Infect Dis 1998;17:479-88.
L Srinivasan, *J Kenneth Department of Laboratory Medicine, Sagar Apollo Hospital, Bangalore - 560 064, Karnataka, India
*Corresponding author (email: <john.kenneth@lvpei.org>) Received: 10-09-05 Accepted: 16-09-05 